\title{
Avaliação familiar e processo de enfermagem: programa de desenvolvimento de competências
}

Family assessment and nursing process: skills and knowledge development program Evaluación familiar y proceso de enfermería: programa de desarrollo de competencias Carolina Miguel Graça Henriques*iD; Eva João de Jesus Santos**(D)

\section{Resumo}

Enquadramento: Na abordagem família é fundamental o enfermeiro deter ferramentas. O Modelo de Calgary de Avaliação Familiar (MCAF), é um instrumento importante na prática de enfermagem familiar.

Objetivos: Conhecer a importância atribuída à família pelos enfermeiros que integram uma unidade de saúde familiar (USF) da Regiáo Centro de Portugal; Identificar os conhecimentos dos enfermeiros sobre avaliaçáo familiar pela aplicação do MCAF; Avaliar o impacto da aplicação de um programa intervenção envolvendo conhecimentos e competências sobre avaliação familiar pelo MCAF nos profissionais de enfermagem.

Metodologia: Estudo longitudinal com 8 enfermeiros, do tipo quase experimental, realizado em dois momentos de recolha de dados, desenho do tipo pré e pós teste, sem grupo de controlo. A amostra, selecionou-se pelo método não probabilístico/acidental/conveniência.

Resultados: Os enfermeiros têm atitudes de suporte à família. Existem diferenças estatísticas significativas, de conhecimentos e competências, na avaliação familiar pelo MCAF, antes e após intervenção.

Conclusão: A implementação de programas interventivos na avaliação familiar pelo MCAF, permite transferência de conhecimentos e desenvolvimento de competências.

Palavras-chave: enfermagem familiar; capacitação; enfermeiros; família

\begin{abstract}
Background: In the family approach, it is essential for nurses to have tools to intervene. The Calgary Family Assessment Model (MCAF) is an important instrument in family nursing practice.

Objectives: To know the importance attributed to the family by nurses who are part of a family health unit (FHU) in the Central Region of Portugal; Identify nurses' knowledge of family assessment by applying the MCAF; To evaluate the impact of the application of an intervention program involving knowledge and skills on family assessment by the MCAF in nursing professionals. Methodology: Longitudinal, quasi-experimental study, performed in two moments of data collection, pre and post test design, without control group, with 8 nurses, selected by the non-probabilistic/accidental/convenience method. Results: Nurses have family supportive attitudes. There are significant statistical differences in knowledge and skills in family assessment by the MCAF before and after intervention.

Conclusion: The implementation of intervention programs in family assessment by the MCAF, allows knowledge transfer and skills development.
\end{abstract}

Keywords: family nursing; training; nurses; family

*Ph.D., Professor Adjunto, Escola Superior Saúde, Instituto Politécnico de Leiria, 2411-901 Leiria, Portugal [carolina henriques@ipleiria.pt]. (1) https://orcid.org/00000002-0904-8057. Contribuição no artigo: orientação da investigação e escrita do artigo **MSc., Enfermeira, Escola Superior Saúde, Instituto Politécnico de Leiria, 2411-901, Leiria, Portugal [Eva.Joao.Santos@chleiria.min-saude.pt]. (D https://orcid.org/00000001-7640-048X. Contribuição no artigo: realização do trabalho de investigação e escrita do artigo.

\section{Resumen}

Marco Contextual: en el enfoque familiar, es esencial que las enfermeras tengan herramientas para intervenir El Modelo de Evaluación Familiar de Calgary (MCAF) es un instrumento importante en la práctica de enfermería familiar.

Objetivos: Conocer la importancia atribuida a la familia por las enfermeras que forman parte de una unidad de salud familiar (FHU) en la Región Central de Portugal; Identificar el conocimiento de las enfermeras sobre la evaluación familiar mediante la aplicación del MCAF; Evaluar el impacto de la aplicación de un programa de intervención que involucra conocimientos y habilidades en la evaluación familiar por parte del MCAF.

Metodología: Estudio longitudinal con 8 enfermeras, cuasiexperimental, realizado en dos momentos de recolección de datos, diseño pre y post prueba, sin grupo de control. Muestra seleccionada por el método no probabilístico/accidental/de conveniencia.

Resultados: las enfermeras tienen actitudes de apoyo familiar. Existen diferencias estadísticas significativas en el conocimiento y las habilidades en la evaluación familiar por parte del MCAF antes y después de la intervención. Conclusión: La implementación de programas de intervención en evaluación familiar por parte del MCAF, permite la transferencia de conocimiento y el desarrollo de habilidades.

Palabras clave: enfermería de la familia; capacitación; enfermeros; familia

Recebido para publicação em: 21.10.19

Aceite para publicação em: 09.12.19

Série IV - n. ${ }^{\circ} 23$ - OUT./NOV./DEZ.2019 


\section{Introduçáo}

Vivemos num mundo de frequentes mudanças, transformaçóes politico-económicas, sociodemográficas e culturais. Estes acontecimentos produziram efeitos no seio familiar, criando alteraçóes na sua tipologia, levando ao aparecimento de novas configuraçóes, sendo exemplo disso a proliferação de famílias monoparentais, dos casais sem filhos, vários tipos de unióes, bem como o aumento do número de pessoas a viverem sós. Porém, apesar destas metamorfoses, as funções de suporte e apoio psicológico ao indivíduo permanece inalterável (Fernandes, 2015). Estas transformações criaram necessidades no sistema familiar. Como tal, a ciência de enfermagem não se dissociou destas carências, de tal forma, que nos últimos 30 anos, com o aparecimento e desenvolvimento da especialidade de enfermagem de saúde familiar, tentou dar resposta a estas novas demandas. No nosso país, a especialidade de enfermagem de saúde familiar, recentemente criada pela Ordem dos Enfermeiros, encontra-se numa fase de grande desenvolvimento.

Para intervir e auxiliar a família a ultrapassar estes novos desafios, é necessário dotar os enfermeiros de meios e ferramentas que possibilitem o conhecimento e compreensão das dinâmicas internas da família. Neste contexto, surge o Modelo de Calgary de Avaliação Familiar (MCAF), como guia orientador da atuação do enfermeiro no trabalho com a família. Muitos dos enfermeiros que atualmente trabalham com famílias nos cuidados de saúde primários não tiveram, na sua formação de base, contacto com instrumentos de avaliação familiar, por outro lado, atendendo ao modelo de aquisição de competências de Benner, a competência dos enfermeiros evolui e transforma-se ao longo do tempo (Benner, 2001). Desta forma, consideramos ser pertinente investigar o impacto de um programa de intervenção no desenvolvimento de conhecimentos e competências na avaliação familiar dos enfermeiros, acreditando que ao adotar uma abordagem à família como foco e não como contexto da prática de cuidados de enfermagem, utilizando como referência o $\mathrm{MCAF}$, os enfermeiros potenciarão ganhos em saúde às famílias por eles cuidadas.

Com esta investigação, pretendemos conhecer a importância atribuída à família pelos enfer- meiros que integram uma Unidade de Saúde Familiar (USF) da Região Centro de Portugal; identificar os conhecimentos dos enfermeiros sobre avaliação familiar pela aplicação do MCAF; avaliar o impacto da aplicação de um programa de intervenção envolvendo conhecimentos e competências sobre avaliação familiar pelo MCAF nos profissionais de enfermagem.

\section{Enquadramento}

Deparamo-nos atualmente com inúmeras definiçôes de família, resultado das diferentes ciências que estudam a família, tais como psicologia, sociologia e economia. Estas têm contribuído para as transformaçóes sofridas pelo conceito de família ao longo dos anos nas diferentes culturas.

"Família refere-se a dois ou mais indivíduos que dependem um do outro para dar apoio emocional, físico e económico. Os membros da família são auto-definidos." (Hanson, 2005, p. 6).

O International Council of Nurses (ICN) define família como: "grupo ou unidade funcional todo coletivo composto por pessoas ligadas por consanguinidade, afinidade, relaçóes emocionais ou legais, sendo a unidade ou o todo considerado um sistema que é maior que a soma das partes" (Conselho Internacional de Enfermeiros, 2015 p.143). Wright e Leahey (2011) defendem, que sobretudo interessa saber e respeitar que família, é quem a pessoa diz que é. De acordo com Ratti, Pereira, e Centa (2005, p. 61) "A família é a intermediária entre o indivíduo e a sociedade, pois é nela que se aprende a perceber o mundo e situar-se nele, passando constantemente por processos de negociaçōes." Apesar dos diferentes conceitos de família, esta diversidade, não dissipa a importância que esta unidade tem no bem-estar dos elementos que a compóem, pois é na família que crescemos, nos sustentamos física, psicológica e socialmente. Enquanto célula da sociedade, as famílias, simultaneamente, definem e refletem o meio onde se integram. As alteraçóes sociais, económicas, políticas, culturais, demográficas e tecnológicas têm levado a que as famílias se transformassem e diversificassem (Dias, 2011). Como referido anteriormente, acontecimentos de várias ordens conduziram ao brotar de novas 
necessidades no seio da família, de modo que a enfermagem não ficou alheia a estes factos, ao longo da sua história. Como a ciência sempre demonstrou a sua preocupação do pensar em familia. Assim sendo, surge a disciplina de enfermagem de saúde familiar.

Para Hanson (2005) a enfermagem de saúde familiar é entendida como

o processo de cuidar das necessidades de saúde das famílias que estão dentro do raio de ação da prática de enfermagem . . podendo ter como objetivo a família como contexto, a família como um todo, a família como um sistema ou a família como um componente da sociedade. (Hanson, 2005, p.8)

Assim, para esta autora o enfermeiro poderá desenvolver vários tipos de abordagem à família. As autoras Wright e Leahey (2011), acrescentam que existem diferentes abordagens de acordo com o grau de perícia do profissional de enfermagem, por um lado os enfermeiros de cuidados gerais conceptualizam a família como contexto ou recurso, enquanto que, os enfermeiros especialistas de enfermagem de saúde familiar devem conceptualizar a família como foco de cuidados.

$\mathrm{Na}$ enfermagem, onde a família é foco dos cuidados, não há uma única teoria ou estrutura conceptual que permita descrever de uma forma adequada as relações da família, pelo que é necessário englobar várias perspetivas (Kaakinen, Coehlo, Hanson, \& Gedaly-Duff, 2010). As estruturas conceptuais de enfermagem e as abordagens que sustentam os fundamentos da enfermagem de família desenvolveram-se a partir de três pilares: as teorias das ciências sociais da família, as da terapia familiar e as teorias de enfermagem (Hanson, 2005).

$\mathrm{Na}$ abordagem à família, em qualquer contexto da prática clínica, o enfermeiro beneficia da aplicação de uma estrutura conceptual clara ou de um mapa da família. Essa estrutura favorece a síntese de dados, permitindo a identificação de pontos fortes da família e problemas da família, esboçando assim, de uma forma esquemática, o plano de tratamento dos mesmos (Wright $\&$ Leahey, 2011). Neste contexto surge o Modelo de Calgary de Avaliação Familiar adaptado a partir do Modelo de Tomm e Sanders (1983), que integra, além dos construtos teóricos da disciplina de enfermagem, a teoria geral dos sistemas, a teoria da comunicação, da mudança, a cibernética e a biologia da cognição, do pós-modernismo e feminismo. Trata-se de uma estrutura multidimensional que compreende três categorias principais: estrutural, de desenvolvimento e funcional, em que cada uma destas categorias se divide em várias subcategorias.

Com este estudo, pretende-se, através de um programa de intervenção que os enfermeiros adquiram e desenvolvam competências no que diz respeito à avaliação familiar, ao seu trabalho diário com as famílias, traduzindo também a teoria de aquisição de competências de Benner (2001) por parte destes profissionais. Esta teoria aplicou o modelo de Dreyfus e Dreyfus, de aquisição de competências à enfermagem, pela realização de um longo estudo de investigação, onde encontrou características diferentes na descrição do mesmo caso clínico, feito por vários enfermeiros com níveis de experiência profissional divergentes, desde uma enfermeira iniciada a uma perita. Na aquisição de competências, esta teórica considera, que a teoria é uma ferramenta essencial para explicar e predizer, permite a elaboração de questôes a análise, orientando o profissional na demanda dos problemas, auxiliando na antecipação das necessidades de cuidados.

No trabalho com as famílias, Wright e Leahey (2011) referem que quando os enfermeiros detêm uma estrutura conceptual clara para a avaliação e intervenção nas famílias, estas podem também começar a considerar as novas competências para entrevistar as famílias, no entanto há que distinguir o que é denominado de habilidades de um conhecimento geral, dos conhecimentos e habilidades de uma prática avançada com a família.

\section{Questóes de investigaçáo/Hipóteses}

Qual é a importância atribuída à família dos enfermeiros que integram uma USF da Região Centro de Portugal?; Quais são os conhecimentos dos profissionais de enfermagem que integram uma USF da Regiáo Centro de Portugal, sobre Avaliação Familiar pela aplicação do MCAF?; Qual é o impacto de um programa de desenvolvimento de conhecimentos sobre a avaliação familiar através do MCAF, nos profissionais de enfermagem que integram uma USF da Região 
Centro de Portugal?; Qual é o impacto de um programa de desenvolvimento de competências sobre a avaliação familiar através do MCAF, nos profissionais de enfermagem que integram uma USF da Região Centro de Portugal?

H1 - Existem diferenças estatisticamente significativas nos conhecimentos sobre avaliação familiar pela aplicação do MCAF, nos enfermeiros que integram uma USF da Região Centro de Portugal, antes e após a implementação de um Programa de Intervenção;

$\mathrm{H} 2$ - Existem diferenças estatisticamente significativas nas competências sobre avaliação familiar pela aplicação do MCAF, dos enfermeiros que integram uma USF da Região Centro de Portugal, antes e após a implementação de um programa de intervençáo.

\section{Metodologia}

Foi realizado um estudo longitudinal a um grupo de oito enfermeiros de uma USF da região centro de Portugal, do tipo quase experimental, em dois momentos de recolha de dados, de desenho do tipo pré teste e pós teste, sem grupo de controlo.

A população deste estudo é constituída pelos enfermeiros que exercem funçóes numa USF da Região Centro de Portugal. A amostra deste estudo, foi selecionada pelo método não probabilístico acidental ou de conveniência. Assim sendo, foram definidos como critérios de inclusão: ser enfermeiro a desempenhar funçóes na USF selecionada que se encontram até à data a exercer, não estando ausentes por licença ou motivos de doença e que aceitem participar voluntariamente no estudo. $\mathrm{O}$ tamanho da amostra, foi determinado pelo facto de no momento da colheita de dados eram os que integravam a USF e aceitaram participar no estudo.

A recolha dos dados foi realizada, através de um questionário autoaplicado preenchido antes e após o programa de intervenção (1 mês depois). O questionário era constituído por quatro partes distintas. A primeira parte do questionário continha sete variáveis, atributo que incidia em dados sociodemográficos e profissionais dos sujeitos do estudo, como idade, sexo, habilitações académicas e profissionais, bem como a experiência profissional. $\mathrm{Na}$ segunda parte do questionário, denominada por avaliação da importância da família nos cuidados de enfermagem, tínhamos como variáveis independentes: a perceção dos enfermeiros sobre a enfermagem de saúde familiar; as perceçóes dos enfermeiros sobre as condiçóes importantes para a prática de enfermagem com as famílias e por último, a importância das famílias nos cuidados de enfermagem, através da aplicação da escala IFCE-AE - A Importância das famílias nos cuidados de Enfermagem - atitudes dos enfermeiros (Oliveira et al., 2011), com cinco opções de resposta, versão esta baseada na Escala Families Importance in Nursing Care - Nurses Attitudes (IFCE-AE) revista por Saveman, Benzein, Engström, e Årestedt (2011). A Escala IFCE está organizada com 26 asserçóes e possui 5 opçóes de resposta segundo uma escala do tipo Likert em que 1 -Discordo completamente; 2 - Discordo; 3 - Nem discordo/Nem concordo; 4 - Concordo; 5. Concordo completamente. As pontuações atribuídas variam entre discordo completamente (1 ponto) a concordo completamente (5 pontos). A pontuação geral da escala resulta da soma de cada item, podendo variar entre 26 e 130 pontos. Uma melhor pontuação final obtida significa uma atitude mais favorável do enfermeiro relativamente à participação da família nos cuidados ao utente. O IFCE-AE está organizado em três dimensões (Tabela 1): Família como parceiro dialogante e recurso de coping (12 itens); Família como recurso dos cuidados de enfermagem (10 itens); e Família como um fardo (4 itens).

Tabela 1

Dimensóes da Escala IFCE-AE

\begin{tabular}{ll}
\hline Dimensóes & Itens \\
\hline Parceiro dialogante e recurso de coping & $4 ; 6 ; 9 ; 12 ; 14 ; 15 ; 16 ; 17 ; 18 ; 19 ; 24$ e 25 \\
Recurso nos cuidados de enfermagem & $1 ; 3 ; 5 ; 7 ; 10 ; 11 ; 13 ; 20 ; 21$ e 22 \\
Família como um fardo & $2 ; 8 ; 23$ e 26 \\
\hline
\end{tabular}


Para analisar a fiabilidade, também denominada por consistência interna de um instrumento, recorreu-se ao cálculo do coeficiente alfa $(\alpha)$ de Cronbach, tendo-se posteriormente procedido à sua comparação com os valores de $\alpha$ de Cronbach obtidos por Saveman et al., (2011) no processo de validação da escala (Tabela 2 ). Através do cálculo do $(\alpha)$ de Cronbach $=0,806$, podemos concluir que temos uma adequada consistência interna do instrumento de medida, uma vez que, segundo Vilelas (2017), um resultado de $\alpha$ entre 0,8 e 0,9 corresponde a um bom grau de consistência interna (Tabela 2).

Tabela 2

Análise da fiabilidade do estudo atual e a comparação com a versão FINC-NA (2011)

\begin{tabular}{lc}
\hline Escalas & $\alpha$ Cronbach \\
\hline FINC-NA, 2011 & 0,920 \\
Estudo realizado & 0,806 \\
\hline
\end{tabular}

Nota. FINC-NA = Escala Families Importance in Nursing Care - Nurses Attitudes.

A terceira parte do nosso questionário, avaliou os conhecimentos dos profissionais sobre o processo de enfermagem aplicado à família e sobre a avaliação familiar pela aplicação do MCAF, obtida pela análise do instrumento, por nós construído, constituído por 24 questóes de resposta fechada. A quarta parte do nosso instrumento visou a avaliação de competências do enfermeiro na avaliaçâao familiar utilizando o MCAF, compostas por afirmaçóes, onde foi solicitado ao formando que se autoavaliasse em relação a 10 afirmaçóes relativas a competências pela aplicação do MCAF. No que diz respeito às questốes éticas, o estudo foi aprovado pelo coordenador da unidade de saúde selecionada e pela Comissão de Ética da Unidade de Investigação em Ciências da Saúde: Enfermagem da Escola Superior de Enfermagem de Coimbra (Parecer No P573/03-2019). Foi autorizada a utilização da Escala IFCE-AE, versão traduzida, adaptada e validada para Portugal por Oliveira et al. (2009), designada por Atitudes dos Enfermeiros Face à Família, tendo sido aplicada uma versão atualizada por sugestão de um dos autores IFCE-AE, com cinco opçóes de resposta. Todos os indivíduos incluídos no estudo assinaram o consentimento informado e esclarecido.

O plano de intervenção contemplou as áreas formativas das variáveis dependentes a serem estudadas. Relativamente à área dos conhecimentos sobre a avaliação familiar através do MCAF, foram desenvolvidos aspetos teóricos de três áreas temáticas, tais como: Processo de Enfermagem aplicado à Família, Modelo de Calgary de Avaliação Familiar e por último, os instrumentos
Genograma e Ecomapa.

No que diz respeito às competências na avaliação familiar pelo MCAF, foram trabalhados aspetos práticos, onde foram aplicados os conceitos teóricos anteriormente abordados, envolvendo o treino no preenchimento da grelha do MCAF, bem como o treino de construção de Ecomapas e Genogramas.

Os métodos utilizados para a realização das formaçóes, foram os expositivos e interativos, tendo sido o programa de intervenção realizado em dois períodos distintos.

Um mês após o términus do programa de intervenção, foi dado a preencher aos enfermeiros o questionário da avaliação pós-formação, envolvendo também um questionário de heteroavaliação de competências desenvolvidas no programa de intervenção.

Os dados reunidos foram tratados estatisticamente no programa informático IBM SPSS Statistics, versão 25.0 para o Windows.

Com o objetivo de testar a normalidade da amostra, foi utilizado o teste Shapiro-Wilks, teste de eleição em situaçóes em que amostra é inferior a 50 elementos, que revelou uma distribuição normal das variáveis dependentes em estudo, nomeadamente a variável dos conhecimentos e a variável das competências dos enfermeiros sobre do processo de enfermagem aplicado à família e sobre a avaliação familiar pela aplicaçáo do MCAF (Tabela 3) o que fomenta a utilização de testes paramétricos, para testar as hipóteses em estudo, tendo sido para tal aplicado o teste $t$-student $(t)$. 
Tabela 3

Teste da Normalidade (Shapiro-Wilk)

\begin{tabular}{|c|c|c|c|c|c|c|c|}
\hline \multirow[b]{3}{*}{ Conhecimentos } & \multirow[b]{3}{*}{ Pré teste } & \multicolumn{3}{|c|}{ Kolmogorov-Smirnov } & \multicolumn{3}{|c|}{ Shapiro-Wilk } \\
\hline & & Statistic & $d f$ & Sig. & Statistic & $d f$ & Sig. \\
\hline & & 0,190 & 0,8 & $0,200^{*}$ & 0,908 & 8 & 0,339 \\
\hline & & \multicolumn{3}{|c|}{ Kolmogorov-Smirnov } & \multicolumn{3}{|c|}{ Shapiro-Wilk } \\
\hline Competências & Pré teste & 0,195 & 8 & $0,200^{*}$ & 0,927 & 8 & 0,486 \\
\hline
\end{tabular}

Nota: $d f=$ degrees of freedom (graus de liberdade); Sig.=significância estatistica. *. Este é um limite inferior da significância verdadeira.

\section{Resultados}

No que se refere às caraterísticas sociodemográficas observamos que a média das idades dos enfermeiros que participaram é de 46,8 anos $(\sigma=$ 5,87). A totalidade da amostra é do sexo feminino num total de oito enfermeiros. Relativamente ao estado civil, verificamos que $75,00 \%(n=6)$ dos enfermeiros, são casados ou vivem em união de facto, sendo que, $12,50 \%(n=1)$ são divorciados e 12,50 (n=1) são solteiros. No que concerne às habilitaçóes académicas, conclui-se que 100\% ( $n$ $=8$ ) dos enfermeiros são licenciados. Em relaçáo à categoria profissional, observamos que $87,50 \%$ $(n=7)$ são enfermeiros de cuidados gerais, sendo que $12,50 \%(n=1)$ são enfermeiros especialistas.
Quando nos debruçamos sobre a experiência profissional dos enfermeiros que participaram na investigação, verificamos a média é de 24,9 anos $(5,11=\sigma)$, com um valor mínimo (Xmín.) de 17 anos e um valor máximo (Xmáx.) de 33 anos de exercício profissional. No que diz respeito à experiência em cuidados de saúde primários da amostra, conclui-se que a média é de 11,3 anos $(5,1=\sigma)$, com um valor mínimo (Xmín.) de 6 anos e um valor máximo (Xmáx.) de 21 anos de exercício nesta área de cuidados.

Relativamente à escala IFCE-AE versão modificada (Tabela 4) a média global obtida foi de 94,75, permite-nos concluir que a nossa amostra, vê e reconhece a importância da família nos cuidados de enfermagem.

Tabela 4

Caracterização da amostra face à escala IFCE-AE versão modificada (2011) e respetivas dimensóes

\begin{tabular}{|c|c|c|c|c|c|c|c|}
\hline & $\begin{array}{l}\text { Média } \\
(\bar{X})\end{array}$ & $\begin{array}{l}\text { Mediana } \\
\quad(\mathrm{Md})\end{array}$ & $\begin{array}{c}\text { Moda } \\
(\mathrm{Mo})\end{array}$ & $\begin{array}{l}\text { Desvio } \\
\text { Padrão } \\
(\sigma)\end{array}$ & $\begin{array}{l}\text { Valor } \\
\text { Mínimo } \\
\text { (Xmín) }\end{array}$ & $\begin{array}{l}\text { Valor } \\
\text { Máximo } \\
\text { (Xmáx.) }\end{array}$ & $N$ \\
\hline $\begin{array}{l}\text { Família como parceiro } \\
\text { dialogante e recurso de coping }\end{array}$ & 47,50 & 48,00 & 47,00 & 4,96 & 39,00 & 53,00 & 8 \\
\hline $\begin{array}{l}\text { Família como recurso nos } \\
\text { cuidados de enfermagem }\end{array}$ & 39,25 & 41,00 & 41,00 & 4,71 & 29,00 & 44,00 & 8 \\
\hline Família como um fardo & 8,00 & 7,5 & 5,00 & 2,67 & 5,00 & 13,00 & 8 \\
\hline Total da IFCE-AE & 94,75 & 96,00 & 96,00 & 7,81 & 77,00 & 102,00 & 8 \\
\hline
\end{tabular}

Nota. Escala IFCE-AE - A Importância das famílias nos cuidados de Enfermagem; $N$ = População Alvo.

Relativamente à avaliação de conhecimentos, na fase inicial, verificou-se que a média foi de 8,88 , indicando assim, que o nível de conhecimentos dos profissionais no momento anterior à intervenção era baixo, num total de classificação de 0 a 24 possível. Na segunda avaliação, a média foi de 22,63, verificando-se uma melhoria acentuada no valor médio traduzindo-se numa melhoria do nível dos conhecimentos dos profissionais nesta variável (Tabela 5). 
Tabela 5

Caracterização da amostra face à avaliação de conhecimentos dos enfermeiros antes e após o programa de intervenção

\begin{tabular}{|c|c|c|c|c|c|c|c|c|}
\hline & & $\begin{array}{l}\text { Média } \\
(\bar{X})\end{array}$ & $\begin{array}{l}\text { Mediana } \\
(\mathrm{Md})\end{array}$ & $\begin{array}{c}\text { Moda } \\
\text { (Mo) }\end{array}$ & $\begin{array}{c}\text { Desvio } \\
\text { Padrão } \\
(\sigma)\end{array}$ & $\begin{array}{c}\text { Valor } \\
\text { Mínimo } \\
\text { (Xmín })\end{array}$ & $\begin{array}{l}\text { Valor } \\
\text { Máximo } \\
\text { (Xmáx.) }\end{array}$ & $N$ \\
\hline Avaliação de & Pré teste & 8,88 & 10,00 & 16,00 & 6,31 & 0,00 & 16,00 & 8 \\
\hline Conhecimentos & Pós teste & 22,63 & 23,00 & 23,00 & 1,06 & 20,00 & 23,00 & 8 \\
\hline
\end{tabular}

Nota. $N$ = População Alvo.

No que respeita à avaliação de competências, na primeira avaliação, o score médio foi de 13,50 , permitindo concluir face aos critérios definidos que o nível médio de competência dos enfermeiros, era insuficiente. No segundo momento de avaliação, através do questionário aplicado, a média foi de 27,94, classificando-se o nível de competências em muito boas (Tabela 6). Concluiu-se que houve um acréscimo considerável ao nível das competências dos enfermeiros no processo de enfermagem à família e sobre a avaliação familiar na aplicação do MCAF, após a intervençáo.

Tabela 6

Caracterização da amostra face à avaliação de competências dos enfermeiros antes e após o programa de intervenção

\begin{tabular}{|c|c|c|c|c|c|c|c|c|}
\hline & & $\begin{array}{l}\text { Média } \\
(\bar{X})\end{array}$ & $\begin{array}{l}\text { Mediana } \\
\text { (Md) }\end{array}$ & $\begin{array}{l}\text { Moda } \\
(\mathrm{Mo})\end{array}$ & $\begin{array}{c}\text { Desvio } \\
\text { Padrão } \\
(\sigma) \\
\end{array}$ & $\begin{array}{c}\text { Valor } \\
\text { Mínimo } \\
\left(X_{\text {mín }}\right)\end{array}$ & $\begin{array}{c}\text { Valor } \\
\text { Máximo } \\
\left(X_{\text {máx. }}\right)\end{array}$ & $N$ \\
\hline $\begin{array}{l}\text { Avaliação de } \\
\text { competências Processo }\end{array}$ & Pré teste & 3,50 & 14,50 & 15,00 & 7,50 & 4,00 & 28,00 & 8 \\
\hline $\begin{array}{l}\text { de Enfermagem à } \\
\text { Família }\end{array}$ & Pós teste & 27,94 & 28,50 & 28,50 & 1,78 & 24,00 & 30,00 & 8 \\
\hline
\end{tabular}

Nota. $N=$ População Alvo.

Para testar as hipóteses deste estudo, utilizámos o teste $t$ de Student, que de acordo com Vilelas (2017) é aplicado a uma variável quantitativa discreta ou contínua que tem uma curva de distribuição normal e em duas amostras relacionadas. H1 - Existem diferenças estatisticamente significativas nos conhecimentos sobre avaliação familiar pela aplicaçáo do MCAF, dos enfermeiros que integram uma USF da Região Centro de Portugal, antes e após a implementação de um Programa de Formação.
No resultado da aplicação do teste $t$ de Student relativamente à avaliação de conhecimentos sobre avaliação familiar pela aplicação do MCAF, verificou-se que existem diferenças estatisticamente significativas $(t=-5,67 ; p \leq 0,05)$ nos conhecimentos entre o segundo momento de avaliação e o primeiro momento de avaliação sobre avaliação familiar pela aplicação do MCAF, nos enfermeiros que integram uma USF da Regiāo Centro de Portugal após o programa de intervenção (Tabela 7). 
Tabela 7

Aplicação do teste estatístico de $t$ de Student para amostras relacionadas face à avaliação de conhecimentos dos enfermeiros de uma USF da região centro do pais entre o momento do pré teste e o momento do pós teste

\begin{tabular}{|c|c|c|c|c|c|c|c|c|}
\hline & \multicolumn{5}{|c|}{ Diferenças emparelhadas } & & & \multirow[b]{3}{*}{$\begin{array}{c}\text { Sig. } \\
\text { (2 extremidades) }\end{array}$} \\
\hline & \multirow[b]{2}{*}{$\begin{array}{l}\text { Média } \\
(\bar{X})\end{array}$} & \multirow[b]{2}{*}{$\begin{array}{c}\text { Desvio } \\
\text { Padrão }(\sigma)\end{array}$} & \multirow[b]{2}{*}{$\begin{array}{l}\text { Desvio padrão } \\
\text { da média }\end{array}$} & \multicolumn{2}{|c|}{$\begin{array}{l}95 \% \text { Intervalo de } \\
\text { Confiança da Diferença }\end{array}$} & \multirow[b]{2}{*}{$t$} & \multirow[b]{2}{*}{$d f$} & \\
\hline & & & & Inferior & Superior & & & \\
\hline $\begin{array}{l}\text { Avaliaçáo de } \\
\text { conhecimentos entre } \\
\text { o momento do pré } \\
\text { teste e o momento } \\
\text { do pós teste }\end{array}$ & $-13,75$ & 6,86 & 2,43 & $-19,49$ & $-8,01$ & $-5,67$ & 7,00 &, 001 \\
\hline
\end{tabular}

Nota. $t=t$ de Student; $d f=$ degrees of freedom (graus de liberdade); Sig.= significância estatística.

H2 - Existem diferenças estatisticamente significativas nas competências sobre avaliação familiar pela aplicação do MCAF, dos enfermeiros que integram uma USF da Regiáo Centro de Portugal, entre o momento do pré teste e o momento do pós teste da implementação de um programa de formação.

No que concerne ao impacto nas competências sobre avaliação familiar pela aplicação do
MCAF, pela aplicação do teste $t$ de Student, observou-se que existem diferenças estatisticamente significativas $(t=-5,01 ; p \leq 0,05)$, entre o momento do pré teste e o momento do pós teste da implementação do programa de intervenção, nas competências sobre avaliação familiar pela aplicação do MCAF, dos enfermeiros que integram uma USF da Região Centro de Portugal (Tabela 8).

Tabela 8

Aplicação do teste estatístico de $t$ de Student para amostras relacionadas face à avaliação de competências dos enfermeiros de uma USF da regiäo centro do país, entre o momento do pré teste e o momento do pós teste

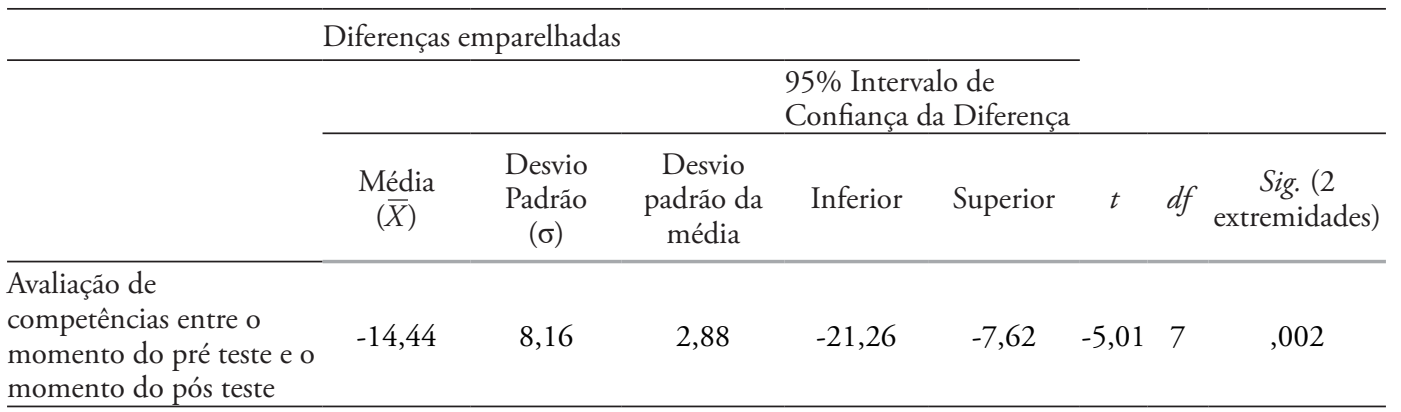

Nota. $\mathrm{t}=t$ de Student; $d f=$ degrees of freedom (graus de liberdade); Sig.= significância estatística.

\section{Discussão}

No que diz respeito às atitudes dos enfermeiros relativamente à importância da família nos cuidados de enfermagem pela aplicação da versão modificada da Escala Importância da Família dos Cuidados de Enfermagem (IFCE-AE) versão modificada, verificou-se que o score médio obtido foi de 94,75, comparando com Saveman et al. (2011), em que a média foi de 104 pontos, os resultados do nosso estudo, ficam ligeiramente abaixo, contudo náo deixa de ser bastante considerável a importância dada à participação da família nos cuidados de enfermagem. Comparando os resultados do nosso estudo com o estudo de Barbieri-Figueiredo et al. (2012), na 
dimensão Família como parceiro dialogante e recurso de coping a média obtida foi de 47,50, ligeiramente inferior a 50,08, do referido estudo, num total de 60 pontos possíveis. A dimensão Família como recurso nos cuidados de enfermagem, a média por nós obtida foi de 39,25, também ligeiramente abaixo do obtido por Barbieri-Figueiredo et al., (2012) que foi de 43,23 pontos num universo de 50 de pontos possíveis. $\mathrm{Na}$ dimensão Família como um fardo, através dos resultados obtidos em que quanto menor a pontuação melhor é atitude dos enfermeiros face à participação nos cuidados de enfermagem, a média obtida foi de 8 , muito próximo do valor de 7,96 alcançado no estudo de Barbieri-Figueiredo et al. (2012) num total de 20 pontos possíveis. Face aos resultados obtidos nas diferentes dimensóes e na globalidade, podemos concluir que os elementos constituintes da amostra possuem atitudes de suporte em relação à família.

No que toca aos conhecimentos da amostra sobre Avaliação familiar através da aplicação do Modelo de Calgary, verificou-se que antes da aplicação do programa de intervenção os mesmos eram baixos com valor médio 8,88 , o que não é motivo de surpresa, pois para Wright e Leahey (2011), a maioria dos enfermeiros na sua formação de base não teve contacto com enfermagem de sistemas. Martins, Fernandes, e Gonçalves (2012), enfatizam que grande parte dos enfermeiros, não conhece qualquer modelo de avaliação ou intervenção na família.

No que concerne ao impacto de um programa de desenvolvimento de conhecimentos sobre a avaliação familiar através do MCAF nos profissionais de enfermagem, salientamos que a média dos conhecimentos pré teste era de 8,88 , e a média pós teste foi de 22. Desta forma, após o tratamento estatístico inferencial, concluiu-se que existem diferenças estatisticamente significativas, nos conhecimentos sobre avaliação familiar pela aplicação do MCAF, dos enfermeiros, antes e após a implementação do programa de intervenção. O programa teve um impacto favorável na aquisição de conhecimentos, o que corrobora com Santos (2012) quando refere que os programas de intervenção educacional neste domínio possibilitam transferência de conhecimento.

Foi também avaliado o impacto de um programa de desenvolvimento de competências sobre a avaliação familiar através do MCAF nos mesmos profissionais. Antes do programa formativo, no que concerne à autoavaliação de competências dos enfermeiros, o score médio foi de 13,50, ou seja nível de competências insuficientes, após a intervenção, a avaliação de competências por heteroavaliação verificou-se que o valor médio obtido, foi de 27,94. Pela aplicação da análise estatística inferencial, concluiu-se existir diferenças estatísticas significativas entre as competências sobre a avaliação familiar através do MCAF, nos enfermeiros participantes, permitindo concluir que advei do desenvolvimento franco de competências com o programa de intervenção - treino no preenchimento da grelha do MCAF, construção de Ecomapas e Genogramas, tal como Fernandes (2014) concluiu no seu estudo ter havido alteração no foco de cuidados, na avaliação e intervençâo da família, bem como, significativo aumento da frequência de registos relativos às categorias e subcategorias do modelo de Calgary.

Como limitaçôes ao estudo, apontamos a escassez de estudos nacionais nesta área, talvez devido à ainda precoce e recentemente criada especialidade de enfermagem, o que faz com que ainda não tenhamos uma elevada produção científica neste domínio, por outro, o facto da nossa amostra ser de tamanho reduzido, não permitindo a generalização dos resultados apresentados.

\section{Conclusáo}

Pela aplicação da versão atualizada da escala a IFCE-AE, verificámos que os enfermeiros possuem atitudes de suporte em relação à família, quer seja na globalidade ou nas suas várias dimensões. Quando testadas as hipóteses de investigação, verificámos que existem diferenças estatisticamente significativas, nos conhecimentos sobre avaliação familiar, pela aplicação do MCAF, dos enfermeiros participantes, antes e após a implementação de um programa de intervenção, confirmando assim a aquisição ou consolidação de conhecimentos, tendo, portanto, impacto favorável ao processo de desenvolvimento de conhecimento.

Também concluímos existir diferenças estatísticas significativas entre as competências sobre a avaliação familiar através do MCAF, nos profissionais de enfermagem que compóem a amostra, levando a concluir que ocorreu desen- 
volvimento de competências com o programa de intervenção.

Com a realização deste estudo podemos dizer que a teoria por si só não é suficiente, é sim, uma ferramenta primordial para explicar e predizer, orientar o enfermeiro a solucionar os problemas, esta servirá de pilar para uma prática competente em enfermagem, na medida em que o conhecimento que envolve a prática, amplia e desenvolve a teoria, adaptando-a à prática dos cuidados de enfermagem. Com este programa de intervençáo pretendeu-se que este sirva de base e trampolim para uma nova abordagem à família. Através das experiências pessoais ou profissionais do trabalho com a família os enfermeiros desenvolvem competências, transitando por vários níveis de competência, divergindo entre eles na forma de pensar, atuar ou intervir.

Face aos resultados alcançados com o programa de intervenção, podemos assumir que é aconselhável a aplicaçáo de planos de intervenção nesta área, contribuindo assim com mais uma achega para o leque de programas formativos e interventivos direcionados a munir os enfermeiros de família de conhecimentos e competências na avaliação familiar pelo modelo de Calgary. Desta forma, pensamos ser possível contribuir para uma melhor prática dos cuidados de enfermagem centrados na família com a replicação deste tipo de intervençáo em outras unidades de saúde.

\section{Referencias bibliográficas}

Barbieri-Figueiredo, M. C., Santos, M. R., Andrade, L., Vilar, A. I., Martinho, M. J., \& Fernandes, I. (2012). Atitudes, conceçóes e praticas dos enfermeiros na prestação de cuidados às famílias em cuidados de saúde primários. In J. C. Carvalho, M. C. Barbieri-Figueiredo, H. I. Fernandes, A. I. Vilar, L. Andrade, M. R. Santos ... P. Oliveira (Coords.), Transferibilidade do conhecimento em enfermagem de família (pp. 36-43). Porto, Portugal: Escola Superior de Enfermagem do Porto.

Benner, P. (2001). De iniciado a perito. Coimbra, Portugal: Quarteto.

Conselho Internacional de Enfermeiros. (2015). CIPE : Classificação Internacional para a Prática de Enfermagem. Lisboa, Portugal: Ordem dos Enfermeiros.

Dias, M. O. (2011). Um olhar sobre a família na perspetiva sistémica - o processo de comunicação no sistema familiar. Gestão e Desenvolvimento, 19, 139-156. doi: 10.7559/gestaoedesenvolvimento.2011.140

Fernandes, C. S. (2014). A família como foco dos cuidados de enfermagem - aprendendo com o "family nursing game" (Tese de doutoramento). Recuperado de https:// sigarra.up.pt/icbas/pt/pub_geral.pub_view?pi_pub_base_id=33839

Fernandes, C. S. (2015). A família como foco dos cuidados enfermagem: Aprendendo com o Family Nursing Game. Loures, Portugal: Lusodidata.

Hanson, S. M. (2005). Enfermagem de cuidados de saúde à família: Teoria, prática e investigação ( $2^{\mathrm{a}} \mathrm{ed}$.). Loures, Portugal: Lusociência.

Kaakinen, J. R., Coehlo, D. P., Hanson, S. M., \& Gedaly-Duff, V. (2010). Family health care nursing: Theory, practice and research ( $4^{\text {th }}$ ed.). Philadelphia, PA: F.A. Davies Company.

Martins, M. M., Fernandes, C. S., \& Gonçalves, L. H. (2012). A família como foco dos cuidados de enfermagem em meio hospitalar: Um programa educativo. Revista Brasileira de Enfermagem, 65(4), 685-690. doi: 10.1590/s0034-71672012000400020

Oliveira, P. C., Fernandes, H. V., Vilar, A. I., Figueiredo, M. H., Ferreira, M. M., Martinho, M. J., . . . Martins, M. M. (2011). Atitudes dos enfermeiros face à família: Validação da escala Families' Importance in Nursing Care - Nurses Attitudes. Revista da Escola de Enfermagem da USP, 45(6), 1331-1337. doi: 10.1590/ S0080-62342011000600008

Ratti, A., Pereira, M., \& Centa, M. (2005). A relevância da cultura no cuidado às famílias. Família, Saúde e Desenvolvimento, 7(1), 60-68. doi: 10.5380/fsd.v7i1.8054

Santos, M. (2012). Abordagem sistémica do cuidado à família: impacto no desempenho profissonal do enfermeiro (Tese de doutoramento). Recuperado de http://hdl.handle. net/10451/6979

Saveman, B.-I., Benzein, E. G., Engström, Å. H., \& Årestedt, K. (2011). Refinement and psychometric reevaluation of the instrument: Families' Importance in Nursing Care - Nurses' Attitudes. Journal of Family Nursing, 17(3), 312-329. doi: 10.1177/1074840711415074

Tomm, K., \& Sanders, G. (1983). Family assessment in a problem oriented record: Diagnosis and assessment in family therapy. London, England: Aspen System Corporation.

Vilelas, J. (2017). Investigação: O processo de construção do conhecimento ( $2^{\mathrm{a}} \mathrm{ed}$.). Lisboa, Portugal: Sílabo.

Wright, L. M., \& Leahey, M. (2011). Enfermeiras e famílias: Guia para avaliação e intervenção na família (5a ed.). São Paulo, Brasil: Roca. 